\title{
The interrelation between premenstrual syndrome and major depression: Results from a population-based sample
}

\author{
Christine Forrester-Knauss ${ }^{1,2^{*}}$, Elisabeth Zemp Stutz ${ }^{1,2}$, Carine Weiss ${ }^{1,2}$ and Sibil Tschudin ${ }^{3}$
}

\begin{abstract}
Background: Research about the relationship between premenstrual syndrome (PMS) and major depression is limited. This study examined the relationship between moderate to severe PMS and major depression in a population-based sample of women of reproductive age. The objectives of the study were to assess the association between premenstrual syndrome and major depression, to analyse how PMS and major depression differ and to characterise the group of women who report both PMS and major depression.

Methods: Data were obtained from the Swiss Health Survey 2007. Included in the analysis was data from women under the age of 55 without hysterectomy and who answered the questions on PMS symptoms. The populationbased sample consisted of 3518 women. Weighted prevalence rates were calculated and relative risk ratios for PMS, major depression and women who reported both PMS and major depression, were calculated with logistic multinominal logit regression.
\end{abstract}

Results: The prevalence of major depression was $11.3 \%$ in women screening positive for moderate PMS and $24.6 \%$ in women screening positive for severe PMS. Compared to women without any of these conditions, women who reported moderate to severe alcohol consumption had a lower risk for PMS. Women reporting use of antidepressants, and use of oral contraceptives had a higher risk for major depression compared to women without any of these conditions. Women reporting work dissatisfaction had a higher risk for PMS. A higher relative risk to report both PMS and major depression compared to women without PMS or major depression was related to factors such as high psychological distress, low mastery, psychotropic drug consumption, and low self-rated health.

Conclusions: The results suggested that women who suffer from both PMS and major depression are more impaired compared to women with only one disorder. The results further indicated that PMS and major depression are different disorders that can, however, co-occur.

Keywords: Premenstrual syndrome, major depression

\section{Background}

Premenstrual syndrome (PMS) and premenstrual dysphoric disorders (PMDD), as a severe form of premenstrual syndrome, have shown to be associated with several psychological conditions, such as reduced psychological wellbeing [1-6], mood disorders, particularly depressive disorders [7-14], and exacerbation of

\footnotetext{
* Correspondence: christine.knauss@unibas.ch

'Department of Epidemiology and Public Health, Swiss Tropical and Public

Health Institute (Swiss TPH), Socinstr. 57, 4051 Basel, Switzerland

Full list of author information is available at the end of the article
}

depression [15]. There is limited research on the relationship between major depression and premenstrual symptoms from studies with large sample sizes or from population-based studies. Wittchen et al. [16] have indicated a high comorbidity between PMDD and other mood disorders $(22.9 \%)$ in a community-based study. Similar comorbidity rates were observed for PMS and major depression by Yonkers et al. [9]. In a U.S. population-based study, it was shown that women with menstrual problems were significantly more likely to report depression [17].

\section{Ciomed Central}


The temporal relationship between PMS and major depression has been investigated in several studies yielding conflicting results. Some studies have shown that women with PMS or PMDD have a higher percentage of past major depression than women without PMS or PMDD $[10,11,18]$, while Hurt et al. have reported contradictory results [19]: Although the risk of late luteal phase dysphoric disorder (LLPDD) (the former term for PMDD in the DSM-III-R) was 14\% higher in women with a past psychological disorder, it was not increased in women who reported to suffer from a major depression in the past [19]. Breaux, Hartlage and Gehlert [20] concluded in their review that based on existing research it has not been fully proven whether women with PMDD have a higher likelihood to report past major depression.

There is also some evidence that women with PMDD might be at a higher risk to develop major depression in the future than women without PMDD [20,21].

Besides investigating the relationship between the two disorders, examination of whether and how the two disorders can be differentiated from each other is also of relevance. For PMDD, the criteria of the DSM-IV require the disturbance not to be merely an exacerbation of the symptoms of another disorder, such as major depression. Nevertheless, a clear distinction between PMDD and depression seems not always as clear as wished, and some symptoms, such as depressed mood, feelings of hopelessness, decreased interest in usual activities, concentration difficulties, lack of energy, change in appetite, hypersomnia or insomnia are included in measures for both disorders [22,23]. Some authors described an overlap of symptoms such as irritability, or mood swings between severe premenstrual syndrome and depression [24]. Others, however, emphasised that despite the intriguing similarities between PMDD and depression, they should be regarded as distinct diagnostic entities [25]. Irritability has been described as a more prominent symptom in women with PMS or PMDD than depressed mood [12,26]. Differences in the dysregulation in the stress axes in women with PMDD and in women with current or past depressive disorders also suggest the two disorders to be distinct [27]. Results of studies about risk factors for depression or PMS suggested that the two disorders might have differing causes. In a longitudinal population-based twin study, it was suggested that genetic and environmental risk factors of premenstrual symptoms and major depression are not closely associated [28]. Premenstrual symptoms seem to be only to a small degree or not at all influenced by familial-environmental factors [28].

To our knowledge, little is known about the group of women who report both major depression and premenstrual symptoms. Comorbidity could be related to higher impairment which would make appropriate treatment for this group of women particularly relevant. Soares et al. [29] have shown that women with PMDD and a history of depression were less educated and reported marital disruption less frequently than women with PMDD and no history of depression.

There is a paucity of data from population-based studies on the association between and the distinction of PMS and major depression, and especially knowledge about the group of women reporting to suffer from both is also limited. The first aim of this study was therefore to assess the prevalence of women reporting both major depression and premenstrual symptoms in a large population-based sample and to analyse how women with PMS and depression differ from each other. A second aim was to characterise women who report both major depression and premenstrual symptoms.

\section{Methods}

\section{Recruitment and Participants}

Data presented in this study was assessed for the nationwide Swiss Health Survey 2007 (SHS), which is conducted every five years since 1992 as a cross-sectional study by the Swiss Federal Office of Statistics. The SHS aims to give information about health status, health related behaviors, prevalence and consequences of diseases, and health care utilization. The data of the SHS are available from the Swiss Federal Office of Statistics upon request. For this study, a permission to analyse the data was obtained (Contract Number 30.23-2008). For the SHS 2007, a random sample of adults living in Switzerland aged 15 years or older was drawn. The assessment consisted of two parts, a telephone interview and an additional written questionnaire. The response rate for the telephone interview was 66\% (18760). The written questionnaire was sent to all participants of the telephone interview. It was completed by $80 \%$ of the participants who had taken part in the telephone interview $(14,432)$. The questions about premenstrual symptoms were included in the written part of the questionnaire.

The sample for this study was restricted to women under the age of 55 with no history of hysterectomy and who answered the questions on PMS symptoms ( $\mathrm{N}=$ 3522). Mean age was 35.46 years.

\section{Assessment of premenstrual syndrome}

To assess premenstrual symptoms and to differentiate between women with or without moderate to severe PMS, a slightly modified version of the premenstrual symptoms screening tool (PSST) [22] was used. The modified PSST version used in this study consists of 10 items (instead of originally 14 items) each describing a 
premenstrual symptom. These items had to be rated on a 4-point Likert scale. The PSST is based on the DSMIV criteria of PMDD. A German translation of the PSST was used for this study. A German version of the PSST by Bentz, Steiner and Meinlschmidt [30] has recently been shown to be a reliable and valid measure for the screening of premenstrual symptoms. Due to restrictions with regard to the number of items that could be included in the Swiss Health Survey, three of the PMS questions were dropped (decreased interest in work activities, home activities and social activities) or merged into one question (insomnia and hypersomnia). The two questions on interference of symptoms with relationships with co-workers and with family were also merged together. To determine severe PMS, the instructions of the premenstrual symptoms screening tool of Steiner et al. [22] were used as followed: For severe PMS, at least one of the main symptoms (anger or irritability, anxiety or tension, tearfulness or mood swings, depressed mood) had to be severe, at least four of the additional symptoms had to be moderate to severe and one of the interferences (work efficiency, relationships with coworkers or family, social life activities, and home responsibilities) had to be severe [22]. For moderate PMS, at least one of the four main symptoms had to be moderate to severe, at least four of the additional symptoms had to be moderate to severe and one of the interferences had to be moderate to severe.

\section{Assessment of major depression}

Major depression was assessed with the World Health Organization's Composite International Diagnostic Interview Short Form (CIDI-SF) [23]. The CIDI-SF is a brief and reliable screening measure that has been validated by Kessler and colleagues [23] and allows generating DSM-IV diagnoses. To calculate a score, 23 of the 35 items of the interview were used. The score was based on reported type and length of symptoms.

\section{Statistical Analyses}

Chi-square tests and multinominal logit regression were conducted using STATA 10 to compare the four groups: women without major depression or PMS, women with major depression only, women with PMS only and women with both major depression and PMS. The statistical analyses were considered to be significant with $\mathrm{p}<$ 0.05. For the chi-square tests weighted dated was used.

\section{Results}

\section{Characteristics of the sample}

Table 1 shows sociodemographic characteristics of the total sample and of the four groups (women without PMS and depression, women with PMS, women with major depression, women with both PMS and depression). No significant differences between the four groups were found in age, but were present in marital status and living arrangements, with the highest proportions of being unmarried and separated/divorced, living alone, and being a single parent in women with major depression. Of the overall sample, $7.2 \%$ had a major depression, 10.3\% moderate PMS and 3.2\% severe PMS.

\section{Relationship and differences in women with PMS and major depression}

Table 2 shows that $11.3 \%$ of women with moderate PMS and $24.6 \%$ of women with severe PMS suffered additionally from major depression compared to $6.2 \%$ of the women who did not report PMS. Current treatment for depression was reported by $3.4 \%$ of the women with moderate PMS and $15.1 \%$ of the women with severe PMS compared to $2.4 \%$ of women without PMS. Daily consumption of antidepressants was highest in women with severe PMS (23.4\%) and lowest in women without PMS (5.8\%). The highest percentage of women taking tranquilizers or sleeping pills was in women with moderate PMS (16.0\%). Of the women with severe PMS, 35\% reported to take any type of psychotropic medication.

Differences in health status and health behaviour between the four groups (women without major depression or PMS, women with PMS, women with major depression, women with major depression plus PMS) are shown in Table 3. They were found to be significant for most of the health status variables. The highest percentage of women taking oral contraception was in the group of women who reported suffering from major depression (37.9\%). A significant difference between the four groups was also found in alcohol consumption ( $\mathrm{p}=$ .006) with $9.2 \%$ of women with major depression reporting moderate to severe consumption compared to $3.6 \%$ of the women without any of the two disorders. Significant differences were also found in psychotropic drug consumption $(\mathrm{p}=.000)$, work satisfaction $(\mathrm{p}=.0009)$, mastery $(\mathrm{p}=.000)$, self-rated health $(\mathrm{p}=.000)$, sleeping difficulties $(\mathrm{p}=.000)$ and psychological distress $(\mathrm{p}=$ .000). Among the four groups, consumption of antidepressants only was highest in women with major depression only $(12.4 \%)$. In contrary, consumption of sleeping pills/tranquillizers and antidepressants was highest in women with both conditions, PMS and major depression (35.8\%). Of the women with both conditions, $74.4 \%$ reported low mastery, $25.8 \%$ poor self-rated health, $33.5 \%$ high psychological distress, $33.6 \%$ strongly agreed to sleeping difficulties, and $27.1 \%$ reported to be partly satisfied to extremely dissatisfied with work.

\section{Predictors for PMS and/or major depression}

Table 4 shows the results of the multinominal logit regression. Relative risk ratios (RRR) were calculated to 
Table 1 Socio-demographic characteristics in women without and with PMS, major depression, and with PMS plus depression (weighted prevalence rates)

\begin{tabular}{|c|c|c|c|c|c|c|}
\hline & $\begin{array}{l}\text { No PMS or depression } \\
\qquad \begin{array}{c}(n=2848) \\
\%\end{array}\end{array}$ & $\begin{array}{c}\text { PMS Only } \\
(n=413) \\
\%\end{array}$ & $\begin{array}{l}\text { Major depression only } \\
\qquad \begin{array}{c}(n=197) \\
\%\end{array}\end{array}$ & $\begin{array}{l}\text { PMS plus depression } \\
\qquad \begin{array}{c}(n=60) \\
\%\end{array}\end{array}$ & $\begin{array}{c}\text { Total } \\
(n=3518) \\
\%\end{array}$ & $\begin{array}{c}\chi^{2} \\
\text { (df) }\end{array}$ \\
\hline \multicolumn{7}{|l|}{ Age (years) } \\
\hline $14-25$ & 23.7 & 24.4 & 23.1 & 24.1 & 23.8 & \\
\hline $24-35$ & 27.5 & 21.2 & 28.2 & 24.4 & 26.7 & \\
\hline $34-45$ & 31.8 & 37.4 & 30.0 & 23.4 & 32.2 & \\
\hline $44-55$ & 17.0 & 17.0 & 18.8 & 28.1 & 17.3 & \\
\hline Total & 100 & 100 & 100 & 100 & 100 & $9.13(9)$ \\
\hline \multicolumn{7}{|l|}{ Marital status } \\
\hline Unmarried & 41.8 & 43.3 & 46.1 & 42.2 & 42.2 & \\
\hline Married & 50.6 & 47.5 & 41.5 & 50.0 & 49.7 & \\
\hline Widowed & 0.6 & 1.3 & 0.7 & 0.0 & 0.7 & \\
\hline Separated/Divorced & 7.0 & 7.9 & 11.8 & 7.9 & 7.3 & \\
\hline Total & 100 & 100 & 100 & 100 & 100 & $18.95^{*}(9)$ \\
\hline \multicolumn{7}{|l|}{ Language regions } \\
\hline German-speaking & 71.3 & 63.6 & 71.6 & 52.5 & 70.1 & \\
\hline French-speaking & 24.4 & 30.2 & 24.4 & 39.8 & 25.4 & \\
\hline Italian-speaking & 4.2 & 6.2 & 4.0 & 7.8 & 4.5 & \\
\hline Total & 100 & 100 & 100 & 100 & 100 & $10.56(6)$ \\
\hline \multicolumn{7}{|l|}{ Nationality } \\
\hline Swiss & 75.9 & 70.5 & 75.0 & 58.7 & 74.9 & \\
\hline Other & 24.1 & 29.5 & 25.0 & 41.3 & 25.1 & \\
\hline Total & 100 & 100 & 100 & 100 & 100 & $4.43(3)$ \\
\hline \multicolumn{7}{|l|}{ Profession } \\
\hline Higher-middle management & 42.5 & 38.6 & 39.5 & 29.6 & 41.7 & \\
\hline Office worker & 31.9 & 39.4 & 29.1 & 23.2 & 32.4 & \\
\hline Craftsperson & 5.5 & 4.9 & 6.8 & 9.7 & 5.5 & \\
\hline Labourer & 20.1 & 17.2 & 24.6 & 37.5 & 20.3 & \\
\hline Total & 100 & 100 & 100 & 100 & 100 & $9.39(9)$ \\
\hline \multicolumn{7}{|l|}{ Education } \\
\hline Compulsory school & 9.3 & 9.0 & 8.0 & 25.8 & 9.5 & \\
\hline Secondary school & 65.3 & 66 & 69.7 & 59.6 & 65.5 & \\
\hline College/university & 25.4 & 25 & 22.4 & 14.6 & 25 & \\
\hline Total & 100 & 100 & 100 & 100 & 100 & $5.86(6)$ \\
\hline \multicolumn{7}{|l|}{ Employment } \\
\hline Full time & 30.1 & 26.6 & 29.0 & 21.6 & 29.4 & \\
\hline Part time & 45.4 & 41.1 & 49.2 & 39.8 & 45 & \\
\hline No employment & 24.5 & 32.3 & 21.8 & 38.5 & 25.5 & \\
\hline Total & 100 & 100 & 100 & 100 & 100 & $7.77(6)$ \\
\hline \multicolumn{7}{|l|}{ Living arrangements } \\
\hline Living alone & 8.8 & 10.0 & 17.0 & 10.1 & 9.4 & \\
\hline Couple without child & 18.9 & 14.9 & 16.5 & 18.7 & 18.3 & \\
\hline Couple with child & 62 & 63.5 & 49.4 & 62.3 & 61.5 & \\
\hline Single parent & 8.5 & 9.7 & 16.2 & 7.4 & 9 & \\
\hline Other & 1.8 & 1.9 & 0.8 & 1.6 & 1.8 & \\
\hline Total & 100 & 100 & 100 & 100 & 100 & $21.78^{*}(12)$ \\
\hline
\end{tabular}

${ }^{*} p<.05$ 
Table 2 Frequencies of major depression, treatment for depression, treatment for psychological problems, consumption of psychotropic medication, and antidepressants in women with and without PMS (weighted prevalence rates)

\begin{tabular}{|c|c|c|c|c|c|}
\hline & $\begin{array}{c}\text { No PMS } \\
(n=3045)\end{array}$ & $\begin{array}{l}\text { Moderate PMS } \\
\quad(n=363)\end{array}$ & $\begin{array}{c}\text { Severe PMDD } \\
\quad(n=110)\end{array}$ & $\begin{array}{c}\text { Total } \\
(n=3518)\end{array}$ & $\chi^{2}(\mathrm{df})$ \\
\hline & $\%$ & $\%$ & $\%$ & $\%$ & \\
\hline \multicolumn{6}{|l|}{ Major Depression } \\
\hline Major Depression & 6.2 & 11.3 & 24.6 & 7.3 & \\
\hline No Major Depression & 93.8 & 88.7 & 75.4 & 92.7 & \\
\hline Total & 100 & 100 & 100 & 100 & $11.58^{* *}(2)$ \\
\hline \multicolumn{6}{|l|}{ Treatment for Depression } \\
\hline No & 92.1 & 84.5 & 73.6 & 90.8 & \\
\hline Yes, before the last 12 months & 3.6 & 7.9 & 6.2 & 4.1 & \\
\hline Yes, within the last 12 months & 1.9 & 4.2 & 5.2 & 2.2 & \\
\hline In treatment at the moment & 2.4 & 3.4 & 15.1 & 2.9 & \\
\hline Total & 100 & 100 & 100 & 100 & $18.02^{* *}(6)$ \\
\hline \multicolumn{6}{|l|}{ Consumption antidepressants } \\
\hline Daily & 5.8 & 9.7 & 23.4 & 7.0 & \\
\hline Once to Several times per week & 0.5 & 0.6 & 8.1 & 0.9 & \\
\hline Never & 93.7 & 89.7 & 68.5 & 92.1 & \\
\hline Total & 100 & 100 & 100 & 100 & $6.77(4)$ \\
\hline \multicolumn{6}{|c|}{ Treatment psychological problems in the last 12 months } \\
\hline Yes & 5.7 & 12.5 & 19.7 & 6.8 & \\
\hline No & 94.3 & 87.5 & 80.3 & 93.2 & \\
\hline Total & 100 & 100 & 100 & 100 & $14.81^{* * *}(2)$ \\
\hline \multicolumn{6}{|l|}{ Consumption of psychotropic medication } \\
\hline None & 88.8 & 73.7 & 65.0 & 85.9 & \\
\hline Antidepressants only & 3.7 & 5.4 & 6.9 & 4.0 & \\
\hline Tranquilizers/sleeping pills only & 4.9 & 16.0 & 3.5 & 6.2 & \\
\hline Tranquilizers/sleeping pills and antidepressants & 2.6 & 4.9 & 24.6 & 3.8 & \\
\hline Total & 100 & 100 & 100 & 100 & $17.50 * *(6)$ \\
\hline
\end{tabular}

${ }^{*} \mathrm{p}<.05 ;{ }^{* *} \mathrm{p}<.01 ;{ }^{* * *} \mathrm{p}<.001$

compare the four groups (women without major depression or PMS, women with PMS, women with major depression, and women with both conditions) with women without major depression or PMS as reference group. Language region (Italian-speaking) and education were the only socio-demographic factors significantly associated with one of the four groups: women from the Italian-speaking part had a higher risk of reporting PMS than the reference group (RRR 1.84, $\mathrm{p}<.01)$. Women with a secondary school (RRR 3.83, $\mathrm{p}<.05$ ) or a university/college degree (RRR 3.72, $\mathrm{p}<.05$ ) also had a higher likelihood of reporting PMS than women of the reference group.

Women taking oral contraception were at higher risk of suffering from major depression than women with none of the two conditions (reference group) (RRR 1.67, $\mathrm{p}<.05)$. Women who reported taking antidepressants were more likely to suffer from depression (RRR 3.16, p $<.01$ ) and from depression and PMS (RRR 5.38, p <
.05). The consumption of both tranquillizers/sleeping pills and antidepressants, was more likely in women with depression and PMS (RRR 5.18, p < .05). Women who reported having moderate to severe alcohol consumption were at lower risk of screening positive for PMS than the reference group (RRR 0.34, $\mathrm{p}<.05$ ). Differing associations in alcohol consumption were found for women with PMS (RRR below 1) and women with major depression (RRR above 1).

Women with medium or high psychological distress showed a higher risk of suffering from major depression (medium distress: RRR 1.68, $\mathrm{p}<.05$; high distress: RRR $3.32, \mathrm{p}<.01)$ and an even higher risk of suffering from PMS plus major depression (high distress: RRR 7.97, p < .001) than women of the reference group. High mastery and good self-rated health were protective factors. Women who reported high mastery had a lower risk of screening positive for PMS (RRR 0.39, p < .001), from major depression (RRR 0.34, $\mathrm{p}<.001$ ) and from both 
Table 3 Health status and health behavior in women without and with PMS, major depression, and with PMS plus major depression (weighted prevalence rates)

\begin{tabular}{|c|c|c|c|c|c|c|}
\hline & $\begin{array}{l}\text { No PMS or depression } \\
\quad(n=2848)\end{array}$ & $\begin{array}{l}\text { PMS only } \\
(n=413)\end{array}$ & $\begin{array}{l}\text { Major depression only } \\
\qquad(n=197)\end{array}$ & $\begin{array}{l}\text { PMS plus depression } \\
\qquad(n=60)\end{array}$ & $\begin{array}{c}\text { Total } \\
(n=3518)\end{array}$ & $\chi^{2}(\mathrm{df})$ \\
\hline & $\%$ & $\%$ & $\%$ & $\%$ & $\%$ & \\
\hline \multicolumn{7}{|l|}{ Body weight } \\
\hline Underweight & 6.9 & 6.9 & 9.7 & 7.8 & 7.0 & \\
\hline Normal weight & 74.5 & 72.8 & 69.2 & 71.8 & 74 & \\
\hline Overweight & 13.5 & 13.3 & 12.3 & 9 & 13.3 & \\
\hline Obese & 5.1 & 7.0 & 8.8 & 11.4 & 5.6 & \\
\hline Total & 100 & 100 & 100 & 100 & 100 & $4.85(9)$ \\
\hline \multicolumn{7}{|l|}{ Pill as oral contraception } \\
\hline No & 68 & 78.4 & 62.1 & 65.4 & 68.8 & \\
\hline Yes & 32 & 21.6 & 37.9 & 34.6 & 31.2 & \\
\hline Total & 100 & 100 & 100 & 100 & 100 & $9.85^{*}(3)$ \\
\hline \multicolumn{7}{|l|}{ Alcohol consumption } \\
\hline No consumption & 33.5 & 35.1 & 19 & 40.5 & 33 & \\
\hline Mild consumption & 62.9 & 62.4 & 71.8 & 53.9 & 63.2 & \\
\hline $\begin{array}{l}\text { Moderate to severe } \\
\text { consumption }\end{array}$ & 3.6 & 2.6 & 9.2 & 5.6 & 3.8 & \\
\hline Total & 100 & 100 & 100 & 100 & 100 & $\begin{array}{l}17.99^{* *} \\
(6)\end{array}$ \\
\hline \multicolumn{7}{|l|}{ Smoking status } \\
\hline None smoker & 59.1 & 51.1 & 47.3 & 50.4 & 57.4 & \\
\hline Former smoker & 15.3 & 22 & 17.9 & 18.5 & 16.3 & \\
\hline Current smoker & 25.6 & 26.9 & 34.8 & 31.1 & 26.3 & \\
\hline Total & 100 & 100 & 100 & 100 & 100 & $12.53(6)$ \\
\hline \multicolumn{7}{|l|}{ Cannabis consumption } \\
\hline Yes & 2.5 & 2.7 & 6.2 & 7.4 & 2.8 & \\
\hline No & 97.5 & 97.3 & 93.8 & 92.6 & 97.2 & \\
\hline Total & 100 & 100 & 100 & 100 & 100 & $4.11(3)$ \\
\hline \multicolumn{7}{|l|}{ Health orientation } \\
\hline Not health oriented & 9.7 & 9.6 & 17 & 9.6 & 10.1 & \\
\hline Health oriented & 90.3 & 90.4 & 83 & 90.4 & 89.9 & \\
\hline Total & 100 & 100 & 100 & 100 & 100 & $4.06(3)$ \\
\hline \multicolumn{7}{|l|}{$\begin{array}{l}\text { Psychotropic drug } \\
\text { consumption }\end{array}$} \\
\hline None & 90.9 & 78.2 & 67.3 & 42.6 & 86 & \\
\hline Antidepressants only & 2.9 & 4.9 & 12.4 & 9.7 & 4 & \\
\hline Tranquillizer/sleeping pills only & 4.6 & 13.1 & 7.5 & 11.9 & 6.2 & \\
\hline $\begin{array}{l}\text { Tranquillizer/sleeping pills and } \\
\text { antidepressants }\end{array}$ & 1.5 & 3.8 & 12.7 & 35.8 & 3.7 & \\
\hline Total & 100 & 100 & 100 & 100 & 100 & $\begin{array}{l}38.99^{* * *} \\
\quad(9)\end{array}$ \\
\hline \multicolumn{7}{|l|}{ Work satisfaction } \\
\hline Extremely satisfied & 23.5 & 13.6 & 16.1 & 12.1 & 21.8 & \\
\hline Very satisfied & 45.2 & 41.1 & 33 & 39.4 & 43.9 & \\
\hline Fairly satisfied & 21.1 & 24.4 & 35.2 & 21.4 & 22.3 & \\
\hline $\begin{array}{l}\text { Partly satisfied to extremely } \\
\text { dissatisfied }\end{array}$ & 10.1 & 20.9 & 15.8 & 27.1 & 12 & \\
\hline Total & 100 & 100 & 100 & 100 & 100 & $\begin{array}{l}28.35^{* * *} \\
(9)\end{array}$ \\
\hline \multicolumn{7}{|l|}{ Mastery } \\
\hline Low & 17.4 & 37.6 & 41 & 74.4 & 22 & \\
\hline Medium & 46.9 & 47.7 & 38.3 & 20.3 & 46.1 & \\
\hline
\end{tabular}


Table 3 Health status and health behavior in women without and with PMS, major depression, and with PMS plus major depression (weighted prevalence rates) (Continued)

\begin{tabular}{|c|c|c|c|c|c|c|}
\hline High & 35.6 & 14.7 & 20.7 & 5.3 & 31.9 & \\
\hline Total & 100 & 100 & 100 & 100 & 100 & $\begin{array}{c}104.00^{* * *} \\
(6)\end{array}$ \\
\hline \multicolumn{7}{|c|}{ Physical activity } \\
\hline Inactive & 11.3 & 16.6 & 12.5 & 8.1 & 11.9 & \\
\hline Partially active & 47.2 & 45.2 & 51 & 56.7 & 47.4 & \\
\hline Active & 41.5 & 38.2 & 36.5 & 35.2 & 40.7 & \\
\hline Total & 100 & 100 & 100 & 100 & 100 & $6.35(6)$ \\
\hline \multicolumn{7}{|c|}{ Self-rated health } \\
\hline Poor health & 0.6 & 3.8 & 5.5 & 25.8 & 1.7 & \\
\hline Fair health & 4.8 & 8.6 & 13.6 & 22.8 & 6.1 & \\
\hline Good health & 94.6 & 87.6 & 80.9 & 51.4 & 92.2 & \\
\hline Total & 100 & 100 & 100 & 100 & 100 & $\begin{array}{c}40.23^{* * * *} \\
(6)\end{array}$ \\
\hline \multicolumn{7}{|c|}{ Sleeping difficulties } \\
\hline Not at all & 66.8 & 53.6 & 49.8 & 31.4 & 63.7 & \\
\hline A bit & 27.2 & 29 & 30.2 & 35.0 & 27.8 & \\
\hline Strongly & 6.0 & 17.4 & 20.0 & 33.6 & 8.6 & \\
\hline Total & 100 & 100 & 100 & 100 & 100 & $\begin{array}{c}46.42^{* * * *} \\
(6)\end{array}$ \\
\hline \multicolumn{7}{|c|}{ Psychological distress } \\
\hline Low & 84.2 & 66.0 & 55.2 & 34.3 & 79.6 & \\
\hline Medium & 12.9 & 23.8 & 30.1 & 32.2 & 15.4 & \\
\hline High & 2.9 & 10.3 & 14.7 & 33.5 & 4.9 & \\
\hline Total & 100 & 100 & 100 & 100 & 100 & $\begin{array}{c}77.31^{* * *} \\
(6)\end{array}$ \\
\hline
\end{tabular}

${ }^{*} \mathrm{p}<.05 ;{ }^{* * \mathrm{p}}<.01 ; * * \mathrm{p}<.001$

(RRR $0.15, \mathrm{p}<.05)$ than women of the reference group. Good self-rated health was highly protective against major depression (RRR 0.08, p < .001) and PMS plus major depression (RRR 0.06, $\mathrm{p}<.001$ ). Dissatisfaction at work was a significant risk for PMS (RRR 2.42, p < $.001)$.

\section{Discussion}

Results of this population-based study showed that there was a considerable percentage of women who reported both moderate PMS and major depression (11\% of women with moderate PMS) or severe PMS and major depression (25\% of women with severe PMS). Women with PMS and women with major depression differed mainly in alcohol consumption, psychotropic drug consumption, oral contraception and work dissatisfaction. Factors that were related with a higher relative risk to report both disorders were high psychological distress, low mastery, psychotropic drug consumption, and low self-rated health.

Differences in women with PMS and major depression Women who reported using oral contraceptives were more likely to suffer from major depression than the reference group (women without major depression or PMS). Although not significant, they were also less likely to screen positive for PMS, which also has been found in other studies [31]. These results reflect the controversial and inconsistent results of studies on the effects of oral contraceptives on mood disorders and PMS [32-36]. In clinical practice, it seems therefore important to be aware of a higher possibility of depression in women who are using oral contraceptives and to consider referring women with depressive symptoms taking oral contraception for further assessment or treatment to psychologists or psychiatrists. Work dissatisfaction was a further factor that showed a differing risk in relation to PMS and major depression. High work dissatisfaction was a specific risk for PMS. Kuczmierczyk et al. [37] also found that women with PMS reported more work pressure compared to women without PMS. The results of this study do not allow concluding if PMS influences work satisfaction or if certain strains at work contribute to experiencing PMS symptoms. In patients reporting work dissatisfaction PMS should be taken into account as an associated factor. A higher consumption of antidepressants in women with major depression was expected. Alcohol was the fourth factor that 
Table 4 Relative risk ratios (95\% confidence intervals) from multinominal logistic regression for women without depression or PMS, women with PMS only, women with major depression only and women with PMS and major depression $(n=2349)$

\begin{tabular}{|c|c|c|c|c|c|c|c|}
\hline & $\begin{array}{c}\text { No major depression } \\
\text { PMS }\end{array}$ & & PMS & & $\begin{array}{c}\text { Major } \\
\text { depression }\end{array}$ & & $\begin{array}{l}\text { PMS and major } \\
\text { depression }\end{array}$ \\
\hline & & RRR & $(95 \% \mathrm{Cl})$ & RRR & $(95 \% \mathrm{Cl})$ & RRR & $(95 \% \mathrm{Cl})$ \\
\hline \multicolumn{8}{|l|}{ Age } \\
\hline $14-24$ & 1 & 1 & & 1 & & 1 & \\
\hline $25-34$ & 1 & 1.14 & $(0.67-1.94)$ & 1.30 & $(0.62-2.69)$ & 1.22 & $(0.30-4.95)$ \\
\hline $35-44$ & 1 & 1.35 & $(0.79-2.3)$ & 1.06 & $(0.49-2.29)$ & 0.52 & $(0.11-2.50)$ \\
\hline $45-54$ & 1 & 1.04 & $(0.56-1.93)$ & 0.96 & $(0.41-2.27)$ & 0.92 & $(0.18-4.71)$ \\
\hline \multicolumn{8}{|l|}{ Language regions } \\
\hline German-speaking & 1 & 1 & & 1 & & 1 & \\
\hline French-speaking & 1 & 1.15 & $(0.86-1.55)$ & 0.74 & $(0.49-1.12)$ & 0.90 & $(0.40-2.03)$ \\
\hline Italian-Speaking & 1 & 1.84 & $\begin{array}{c}(1.18-2.89) \\
* *\end{array}$ & 0.43 & $(0.17-1.07)$ & 0.76 & $(0.17-3.33)$ \\
\hline \multicolumn{8}{|l|}{ Marital status } \\
\hline Unmarried & 1 & 1 & & 1 & & 1 & \\
\hline Married & 1 & 0.72 & $(0.46-1.11)$ & 1.38 & $(0.73-2.61)$ & 1.08 & $(0.33-3.50)$ \\
\hline Widowed & 1 & 0.31 & $(0.04-2.40)$ & 1.07 & $(0.13-9.02)$ & + & + \\
\hline Separated/Divorced & 1 & 0.90 & $(0.54-1.50)$ & 1.26 & $(0.65-2.46)$ & 2.00 & $(0.47-8.54)$ \\
\hline \multicolumn{8}{|l|}{ Nationality } \\
\hline Swiss & 1 & 1 & & 1 & & 1 & \\
\hline Other & 1 & 1.08 & $(0.73-1.62)$ & 1.44 & $(0.84-2.48)$ & 1.77 & $(0.68-4.61)$ \\
\hline \multicolumn{8}{|l|}{ Education } \\
\hline Compulsory school & 1 & 1 & & 1 & & 1 & \\
\hline Secondary school & 1 & 3.83 & $\begin{array}{c}(1.36-10.83) \\
*\end{array}$ & 1.72 & $(0.62-4.74)$ & 0.73 & $(0.17-3.06)$ \\
\hline College/university & 1 & 3.72 & $\begin{array}{c}(1.30-10.68) \\
*\end{array}$ & 1.51 & $(0.53-4.30)$ & 0.68 & $(0.15-3.20)$ \\
\hline \multicolumn{8}{|l|}{ Living arrangements } \\
\hline Living alone & 1 & 1 & & 1 & & 1 & \\
\hline Couple without child & 1 & 0.90 & $(0.57-1.44)$ & 0.56 & $(0.30-1.06)$ & 3.07 & $(0.86-10.97)$ \\
\hline Couple with child & 1 & 1.29 & $(0.81-2.04)$ & 0.58 & $(0.30-1.12)$ & 1.92 & $(0.49-7.51)$ \\
\hline Single parent & 1 & 0.96 & $(0.56-1.65)$ & 1.20 & $(0.62-2.30)$ & 0.85 & $(0.15-4.70)$ \\
\hline Other & 1 & 1.52 & $(0.53-4.33)$ & + & + & 3.77 & $(0.31-45.4)$ \\
\hline \multicolumn{8}{|l|}{ Pill as oral contraception } \\
\hline No & 1 & 1 & & 1 & & 1 & \\
\hline Yes & 1 & 0.67 & $(0.44-1.00)$ & 1.67 & $(1.03-2.72)^{*}$ & 1.10 & $(0.39-3.15)$ \\
\hline \multicolumn{8}{|l|}{ Alcohol consumption } \\
\hline No consumption & 1 & 1 & & 1 & & 1 & \\
\hline Mild consumption & 1 & 0.78 & $(0.58-1.04)$ & 1.32 & $(0.84-2.06)$ & 0.96 & $(0.41-2.27)$ \\
\hline Moderate to severe consumption & 1 & 0.34 & $(0.13-0.88)^{*}$ & 1.87 & $(0.79-4.44)$ & 1.30 & $(0.22-7.51)$ \\
\hline \multicolumn{8}{|l|}{ Psychotropic drug consumption } \\
\hline None & 1 & 1 & & 1 & & 1 & \\
\hline Antidepr. only & 1 & 1.10 & $(0.43-2.79)$ & 3.16 & $(1.40-7.13)^{* *}$ & 5.38 & $(1.40-20.72)^{*}$ \\
\hline Tranquillizer/sleeping pills only & 1 & 1.85 & $(0.95-3.59)$ & 0.70 & $(0.24-2.06)$ & 1.86 & $(0.40-8.62)$ \\
\hline $\begin{array}{l}\text { Tranquillizer/sleeping pills and } \\
\text { antidepressants }\end{array}$ & 1 & 1.18 & $(0.34-4.10)$ & 2.91 & $(0.95-8.90)$ & 5.18 & $(1.13-23.84)^{*}$ \\
\hline \multicolumn{8}{|l|}{ Psychological distress } \\
\hline Low & 1 & 1 & & 1 & & 1 & \\
\hline Medium & 1 & 1.23 & $(0.86-1.77)$ & 1.68 & $(1.05-2.69)^{*}$ & 2.33 & $(0.89-6.10)$ \\
\hline High & 1 & 1.77 & $(0.97-3.23)$ & 3.32 & $(1.67-6.57)^{* *}$ & 7.97 & $(2.64-24.01)^{* * *}$ \\
\hline \multicolumn{8}{|l|}{ Mastery } \\
\hline Low & 1 & 1 & & 1 & & 1 & \\
\hline Medium & 1 & 0.87 & $(0.63-1.19)$ & 0.51 & $(0.33-0.79)^{* *}$ & 0.42 & $(0.17-0.99)^{*}$ \\
\hline
\end{tabular}


Table 4 Relative risk ratios ( $95 \%$ confidence intervals) from multinominal logistic regression for women without depression or PMS, women with PMS only, women with major depression only and women with PMS and major depression $(\mathbf{n}=\mathbf{2 3 4 9}$ ) (Continued)

\begin{tabular}{|c|c|c|c|c|c|c|c|}
\hline High & 1 & 0.39 & $\begin{array}{c}(0.26-0.60) \\
* * *\end{array}$ & 0.34 & $(0.19-0.61)^{* * *}$ & 0.15 & $(0.03-0.73)^{*}$ \\
\hline \multicolumn{8}{|l|}{ Self-rated health } \\
\hline Poor health & 1 & 1 & & 1 & & 1 & \\
\hline Fair health & 1 & 0.81 & $(0.16-4.09)$ & 0.16 & $(0.04-0.64)^{*}$ & 0.18 & (0.03-1.05) \\
\hline Good health & 1 & 0.46 & $(0.10-2.16)$ & 0.08 & $(0.02-0.28)^{* * *}$ & 0.06 & $(0.01-0.33)^{* *}$ \\
\hline \multicolumn{8}{|l|}{ Sleeping difficulties } \\
\hline Not at all & 1 & 1 & & 1 & & 1 & \\
\hline A bit & 1 & 1.25 & $(0.93-1.69)$ & 1.25 & $(0.82-1.89)$ & 1.27 & $(0.54-2.99)$ \\
\hline Strongly & 1 & 1.03 & $(0.62-1.73)$ & 1.41 & $(0.75-2.65)$ & 0.68 & $(0.20-2.29)$ \\
\hline Work satisfaction & 1 & & & & & & \\
\hline Extremely satisfied & 1 & 1 & & 1 & & 1 & \\
\hline Very satisfied & 1 & 1.19 & $(0.80-1.76)$ & 0.84 & $(0.50-1.4)$ & 0.78 & $(0.25-2.48)$ \\
\hline Fairly satisfied & 1 & 1.64 & $(1.07-2.51)^{*}$ & 1.38 & $(0.8-2.38)$ & 0.67 & $(0.18-2.46)$ \\
\hline Partly satisfied to extremely dissatisfied & 1 & 2.42 & $\begin{array}{c}(1.51-3.87) \\
* * *\end{array}$ & 1.05 & $(0.54-2.04)$ & 1.70 & $(0.51-5.63)$ \\
\hline
\end{tabular}

$+:$ Not enough cases in this category to analyze; ${ }^{*} p<.05 ;{ }^{* *} p<.01 ;{ }^{* * *} p<.001$

differentiated between women with PMS and women with major depression. Women with moderate to severe alcohol consumption were less likely to report PMS.

\section{Prevalence of both disorders}

The prevalence of women who reported both disorders was comparable with prevalence rates found by Wittchen et al. [16], with slightly more than $20 \%$ of the women reporting both disorders. Women who reported suffering from severe PMS showed the highest percentage of major depression, with a significant association between severe PMS and major depression.

The results suggest that the group of women who suffered from both PMS and major depression, were the most impaired group, with women with high psychological distress and consumption of antidepressants or a combination of psychotropic drugs having the highest risk to report both conditions. The high impairment and high psychological distress suggests that for treatment an interdisciplinary approach including psychologists or psychiatrists might be beneficial. Good self-rated health and high mastery were protective factors against both conditions (PMS and major depression together). Soares et al. [29] found that women with PMDD and a history of depression were less educated and reported marital disruption less frequently than women with PMDD and no history of depression. These results were not confirmed in this study. Neither marital status nor educational training was significantly associated with both conditions being prevalent.

When considering the discussion about gender differences in depression, the findings about the association between PMS and depression are also of significance. It has been suggested that reproductive hormones might contribute to the increased risk in women of suffering from depression compared to men [38]. The relatively high prevalence of women reporting both PMS and major depression, as found in our study, sustains the hypothesis of the contribution of reproductive hormones and thus might be an explanatory factor for the higher prevalence of depression in women compared to men.

\section{Strengths and limitations}

A limitation of our study is the cross-sectional design. The results can therefore only be interpreted as associations. No causal relationships can be deduced and the history of the development of symptoms is unclear. We can therefore not distinguish if premenstrual symptoms became more severe due to a major depression or if depressive symptoms got worse due to premenstrual symptoms. Furthermore, we used self-reporting measures which might result in an underreporting of the proportion of women with major depression. Assuming that women with major depression tend not to take part in surveys and may be underrepresented in our sample, the prevalence of major depression and of women reporting both disorders may be lower in our study than in the population. The associations between the predictors and the groups may as a result be rather conservative. However, we do not expect a reporting bias between the two affected groups given the observed pattern of associations. The gold standard for the assessment of PMS and PMDD is prospective daily rating. The cross-sectional design and the used screening tools did, however, not allow this procedure and the validity of the diagnosis PMS in our study is therefore not 
entirely clear. For this reason, we did not to use the term PMDD, but the terms moderate and severe PMS. Furthermore, without prospective daily rating it is also not clear whether some of the women reporting PMS suffered from premenstrual exacerbation of major depression and might therefore falsely be included in the group of women with PMS. Strengths of our study were the large population-based sample as well as the use of two standardized screening tools in the Swiss Health Survey allowing us to address their relationship with additional indicators of health status and health behaviour, and to control for confounds.

\section{Conclusions}

The results of this study suggest that for the group of women who suffer from both major depression and PMS, treatment might be particularly important and needed given that they have been found to be more impaired than women with one condition only. The risk for low self-rated health was highest in women with both conditions, which indicates that they are not only impaired on a psychological level but also feel physically unwell. As Breaux et al. [20] have stated in their review, more research needs to be done on the comorbidity between major depression and PMDD. The necessary differentiation between the two disorders to assess comorbidity is challenging and comorbidity might therefore not always be detected and adequately treated in clinical practice. However, results of this study suggest that it would be important to do so, to be able to know more about their co-occurrence and offer adequate treatment.

\section{Acknowledgements}

We thank Dr. Christian Schindler for the statistical support.

\section{Author details}

'Department of Epidemiology and Public Health, Swiss Tropical and Public Health Institute (Swiss TPH), Socinstr. 57, 4051 Basel, Switzerland. ${ }^{2}$ University of Basel, Petersplatz 1, 4003 Basel, Switzerland. ${ }^{3}$ Department of Obstetrics and Gynaecology, University Hospital Basel, Spitalstrasse 21, 4031 Basel, Switzerland.

\section{Authors' contributions}

CFK undertook the statistical analysis, did the literature searches, and wrote the draft of the manuscript. CW contributed to the writing. EZS and ST contributed to the interpretation of the results, the writing, finalizing of the manuscript and provided additional literature. All authors have read and approved the final manuscript.

\section{Competing interests}

The authors declare that they have no competing interests.

Received: 27 December 2010 Accepted: 12 October 2011 Published: 12 October 2011

\section{References}

1. Brown MA, Lewis LL: Cycle-phase changes in perceived stress in women with varying levels of premenstrual symptomatology. Research in Nursing \& Health 1993, 16(6):423-9.
2. Lustyk MK, Widman L, Paschane A, Ecker E: Stress, quality of life and physical activity in women with varying degrees of premenstrual symptomatology. Women Health 2004, 39(3):35-44.

3. Hourani LL, Yuan HX, Bray RM: Psychosocial and lifestyle correlates of premenstrual symptoms among military women. Journal of Womens Health 2004, 13(7):812-821.

4. Rosen LN, Moghadam LZ, Endicott J: Relationship between Premenstrual Symptoms and General Well-Being. Psychosomatics 1990, 31(1):47-54.

5. Woods NF, Lentz MJ, Mitchell ES, Heitkemper M, Shaver J, Henker R: Perceived stress, physiologic stress arousal, and premenstrual symptoms: Group differences and intra-individual patterns. Research in Nursing \& Health 1998, 21(6):511-523.

6. Tschudin S, Bertea PC, Zemp E: Prevalence and predictors of premenstrual syndrome and premenstrual dysphoric disorder in a population-based sample. Arch Womens Ment Health 2010, 13(6):485-494.

7. Lane T, Francis A: Premenstrual symptomatology, locus of control, anxiety and depression in women with normal menstrual cycles. Arch Womens Ment Health 2003, 6(2):127-38.

8. Morse CA, Dennerstein L, Varnavides K, Burrows GD: Menstrual cycle symptoms: comparison of a non-clinical sample with a patient group. $J$ Affect Disord 1988, 14(1):41-50.

9. Yonkers KA, Pearlstein T, Rosenheck RA: Premenstrual disorders: bridging research and clinical reality. Arch Womens Ment Health 2003, 6(4):287-92.

10. Bancroft J, Rennie D, Warner P: Vulnerability to Perimenstrual Mood Change - the Relevance of a Past History of Depressive Disorder. Psychosomatic Medicine 1994, 56(3):225-231.

11. Cohen LS, Soares CN, Otto MW, Sweeney BH, Liberman RF, Harlow BL: Prevalence and predictors of premenstrual dysphoric disorder (PMDD) in older premenopausal women - The Harvard Study of Moods and Cycles. Journal of Affective Disorders 2002, 70(2):125-132.

12. Angst J, Sellaro R, Stolar M, Merikangas KR, Endicott J: The epidemiology of perimenstrual psychological symptoms. Acta Psychiatrica Scandinavica 2001, 104(2):110-116.

13. Warner P, Bancroft J, Dixson A, Hampson M: The Relationship between Perimenstrual Depressive Mood and Depressive-Illness. Journal of Affective Disorders 1991, 23(1):9-23.

14. Payne $J$, Roy PS, Murphy-Eberenz K, Weismann MM, Swartz KL, McInnis MG, Nwulia E, Mondimore FM, MacKinnon DF, Miller EB, Nurnberger JI, Levinson DF, DePaulo JR Jr, Potash JB: Reproductive cycleassociated mood symptoms in women with major depression and bipolar disorder. J Affect Disord 2007, 99(1-3):221-9.

15. Endicott J: The Menstrual-Cycle and Mood Disorders. Journal of Affective Disorders 1993, 29(2-3):193-200.

16. Wittchen HU, Becker E, Lieb R, Krause P: Prevalence, incidence and stability of premenstrual dysphoric disorder in the community. Psychological Medicine 2002, 32(1):119-132.

17. Strine TW, Chapman DP, Ahluwalia IB: Menstrual-related problems and psychological distress among women in the United States. Journal of Womens Health 2005, 14(4):316-323.

18. Critchlow DG, Bond AJ, Wingrove J: Mood disorder history and personality assessment in premenstrual dysphoric disorder. J Clin Psychiatry 2001, 62(9):688-93.

19. Hurt SW, Schnurr PP, Severino SK, Freeman EW, Gise LH, Riveratovar A Steege JF: Late Luteal Phase Dysphoric Disorder in 670 Women Evaluated for Premenstrual Complaints. American Journal of Psychiatry 1992, 149(4):525-530.

20. Breaux C, Hartlage S, Gehlert S: Relationships of premenstrual dysphoric disorder to major depression and anxiety disorders: a re-examination. Journal of Psychosomatic Obstetrics and Gynecology 2000, 21(1):17-24.

21. Hartlage SA, Arduino KE, Gehlert S: Premenstrual dysphoric disorder and risk for major depressive disorder: A preliminary study. Journal of Clinical Psychology 2001, 57(12):1571-1578.

22. Steiner $M$, Macdougall $M$, Brown $E$ : The premenstrual symptoms screening tool (PSST) for clinicians. Archives of Women's Mental Health 2003, 6(3):203-209

23. Kessler RC, Andrews G, Mroczek D, Ustun B, Wittchen H-U: The World Health Organization Composite International Diagnostic Interview shortform (CIDI-SF). International Journal of Methods in Psychiatric Research 1998, 7(4):171-185.

24. Soares CN, Cohen LS: Association between premenstrual syndrome and depression. Journal of Clinical Psychiatry 2000, 61(9):677-677. 
25. Landen M, Eriksson E: How does premenstrual dysphoric disorder relate to depression and anxiety disorders? Depression and Anxiety 2003, 17(3):122-129.

26. Hartlage SA, Arduino KE: Toward the content validity of premenstrual dysphoric disorder: do anger and irritability more than depressed mood represent treatment-seekers' experiences? Psychol Rep 2002, 90(1):189-202.

27. Klatzkin RR, Lindgren ME, Forneris CA, Girdler SS: Histories of major depression and premenstrual dysphoric disorder: Evidence for phenotypic differences. Biol Psychol 2010, 84(2):235-47.

28. Kendler KS, Karkowski LM, Corey LA, Neale MC: Longitudinal populationbased twin study of retrospectively reported premenstrual symptoms and lifetime major depression. American Journal of Psychiatry 1998, 155(9):1234-1240,

29. Soares CN, Cohen LS, Otto MW, Harlow BL: Characteristics of women with premenstrual dysphoric disorder (PMDD) who did or did not report history of depression: a preliminary report from the Harvard Study of Moods and Cycles. J Womens Health Gend Based Med 2001, 10(9):873-8.

30. Bentz D, Steiner M, Meinlschmidt G: SIPS - Screening-Instrument fur pramenstruelle Symptome*: Die deutsche Version des Premenstrual Symptoms Screening Tool zur Erfassung klinisch relevanter Beschwerden. SIPS - screening instrument for premenstrual symptoms*: The German version of Premenstrual Symptoms Screening Tool to assess clinically relevant disturbances.]. Nervenarzt 2011.

31. Warner P, Bancroft J: Factors related to self-reporting of the premenstrual syndrome. Br J Psychiatry 1990, 157:249-60.

32. Oinonen KA, Mazmanian D: To what extent do oral contraceptives influence mood and affect? J Affect Disord 2002, 70(3):229-40.

33. Graham CA, Sherwin BB: The relationship between retrospective premenstrual symptom reporting and present oral contraceptive use. J Psychosom Res 1987, 31(1):45-53.

34. Andersch B, Hahn L: Premenstrual complaints II. Influence of oral contraceptives. Acta Obstet Gynecol Scand 1981, 60(6):579-83.

35. Bancroft J, Rennie D: The impact of oral contraceptives on the experience of perimenstrual mood, clumsiness, food craving and other symptoms. J Psychosom Res 1993, 37(2):195-202.

36. Lopez LM, Kaptein AA, Helmerhorst FM: Oral contraceptives containing drospirenone for premenstrual syndrome. Cochrane Database Syst Rev 2009, , 2: CD006586.

37. Kuczmierczyk AR, Labrum AH, Johnson CC: Perception of family and work environments in women with premenstrual syndrome. Journal of Psychosomatic Research 1992, 36(8):787-795.

38. Burt VK, Stein K: Epidemiology of depression throughout the female life cycle. J Clin Psychiatry 2002, 63(Suppl 7):9-15.

Pre-publication history

The pre-publication history for this paper can be accessed here: http://www.biomedcentral.com/1471-2458/11/795/prepub

doi:10.1186/1471-2458-11-795

Cite this article as: Forrester-Knauss et al:: The interrelation between premenstrual syndrome and major depression: Results from a population-based sample. BMC Public Health 2011 11:795.

\section{Submit your next manuscript to BioMed Central and take full advantage of:}

- Convenient online submission

- Thorough peer review

- No space constraints or color figure charges

- Immediate publication on acceptance

- Inclusion in PubMed, CAS, Scopus and Google Scholar

- Research which is freely available for redistribution

Submit your manuscript at www.biomedcentral.com/submit
Biomed Central 